DOI: $10.3901 / J M E .2019 .12 .050$

\title{
基于张量分解的滚动轴承复合故障 多通道信号降噪方法研究*
}

\author{
胡超凡 1,2 王衍学 ${ }^{2}$ \\ (1. 桂林电子科技大学机电工程学院 桂林 541004; \\ 2. 北京建筑大学城市轨道交通车辆服役性能保障重点实验室 北京 100044)
}

\begin{abstract}
摘要: 针对滚动轴承多通道信号同时滤波的问题, 提出一种新的基于张量分解的多维降噪技术。该方法在高维空间中将时域 信号、频率和通道建模为一个三阶张量模型, 首先通过 Tucker3 分解张量模型, 然后通过 $\mathrm{L}$ 曲线准则来选取截断高阶奇异值 分解(Truncated HOSVD)的截断参数, 根据截断参数求解张量模型, 最后根据原张量的组建方式通过逆变换得到新的目标张 量。通过滚动轴承复合故障仿真分析对所提出的滚动轴承复合故障检测技术的性能进行了评价, 然后应用该方法对轴承试验 台采集的振动信号进行降噪分析, 试验结果表明该方法在滚动轴承复合故障多维降噪以及特征提取中的有效性和可行性。基 于张量分析的多维信号滤波技术将拓宽大数据时代处理异构、多维数据的视野。
\end{abstract}

关键词: 张量分解; 复合故障诊断; 截断高阶奇异值分解; 多通道降噪; 滚动轴承

中图分类号: TH133

\section{Research on Multi-channel Signal Denoising Method for Multiple Faults Diagnosis of Rolling Element Bearings Based on Tensor Factorization}

\author{
HU Chaofan ${ }^{1,2}$ WANG Yanxue ${ }^{2}$
}

(1. School of Mechanical and Electrical Engineering, Guilin University of Electronic Technology, Guilin 541004;

2. Beijing Key Laboratory of Performance Guarantee on Urban Rail Transit Vehicles, Beijing University of Civil Engineering and Architecture, Beijing 100044)

\begin{abstract}
Aiming at simultaneously multi-channel signal filtering and multiple faults diagnosis of the roller bearings, a novel multi-channel signal denoising technique is proposed based on tensor factorization. The original vibration signals are first formulated as a 3-way tensor via temporal signal, frequency and channel in a high dimensional space. The tensor model is factorized using Tucker-3 decomposition. Then, key parameters used in the truncated HOSVD are obtained according to the $L$-curve criterion. Finally, tensor model is solved by those selected truncated parameters, while target tensor is reconstructed by inverse transformation. The performance of the developed technique in detecting faults of roller bearings has been thoroughly evaluated through simulation signals. The presented approach is then applied to reduce noise in vibration signal collected on bearing test-rigs. Experimental results showed that the feasibility and validity of the proposed method in multiple faults detection of the bearings. Multidimensional signal filtering based on tensor will broaden the view in dealing with heterogeneous and multiaspect data in an age of big data.
\end{abstract}

Key words: tensor factorization; multiple faults diagnosis; truncated HOSVD; multi-channel denoising; rolling element bearing

\section{0 前言}

滚动轴承是旋转机械中的关键部件之一，其运 行状态直接关系到整个机械系统的性能。然而由于

* 国家自然科学基金(51875032，51475098，61463010)、广西自然科学杰出 青年基金(2016GXNSFFA380008)和桂林电子科技大学研究生教育创新计 划(2018YJCXB01)资助项目。20180619 收到初稿, 20190302 收到修改稿
重载、高速等恶劣工况，这些关键部件不可避免地 会出现各种故障，可能导致停机，甚至引发灾难性 事故。因此, 在过去几十年中, 为了保证高度的可 用性、可靠性和操作安全性, 高效、可靠的滚动轴 承故障检测技术已经引起了很多研究者的关注 ${ }^{[1-3]}$ 。 振动信号分析由于其测量简单，与结构动力学相关 性高, 因此它是最常用的分析方法 ${ }^{[4]}$ 。然而, 采集 的滚动轴承振动信号实际上是一种复杂的和非平稳 
的调制信号, 并且滚动轴承的故障特征通常被湮没 在随机噪声中 ${ }^{[5]}$ 。为此, 提出的多种信号处理技术 已经被成功用于滚动轴承单通道单故障诊断中。例 如, 基于 VMD 和总变差去噪(TV-MM) ${ }^{[5]}$, 自适应 形态更新小波 ${ }^{[6]}$, 双树复小波 ${ }^{[1]}$ 以及谱峭度 ${ }^{[7]}$ 等都被 成功用于分析轴承故障振动信号。此外, 还提出了 基于人工智能技术的轴承故障诊断方法, 如自适应 密度峰搜索 ${ }^{[8]}$ 。

现有的针对单通道的滚动轴承信号检测技术大 多集中在外圈、内圈或滚动体上的单一缺陷诊断上, 然而实际中多种故障可能共存。目前监测诊断系统 中由于诊断的装备测点多、数据采样频率高, 装备 服役历时长, 所以获取了海量的诊断数据, 推动故 障诊断领域进入了 “大数据” 时代 ${ }^{[9]}$ 。然而, 这些 信号处理技术难以同时处理多通道复合故障振动信 号。因此, 需要研究出一种能够同时检测多通道滚 动轴承复合故障振动信号的降噪技术。

大数据时代背景下, 将不可避免的要在高维空 间中进行多通道以及海量数据的处理。张量分解是 一种非常强大和灵活的数据处理工具, 可以计算模 拟各种各样的多维数据。张量是向量和矩阵的高阶 概化, 为已有的具有自然多维结构的真实数据提供 了有用的表示形式 ${ }^{[10]}$ 。张量分解已经被成功用于面 部识别 ${ }^{[11]}$ 以及旋转机械单通道单故障诊断 ${ }^{[12]}$ 。

张量分解的两种经典模型是 PARAFAC 分解和 Tucker 分解, 与 CP 分解相比较, Tucker 分解是一 种更灵活的张量模型, 这种灵活性是由于它的核心 张量允许元素之间相互交换, 比起一个严格的 PARAFAC 模型, Tucker 分解的核心张量可以使我 们更好地探究多维数据的张量结构。张量分解非常 适合处理多维数据, 本文通过采用时域信号、频率 和通道将滚动轴承复合故障信号建立成三阶 Tucker 张量模型, 并且结合截断高阶奇异值分解(Truncated HOSVD), 提出一种新的滚动轴承复合故障多通道 降噪技术, 同时对滚动轴承复合故障的多个通道进 行滤波, 提高检测效率。通过仿真信号和实际故障 信号试验充分验证了所提出方法的有效性。

\section{1 张量分解的基本理论}

张量 ${ }^{[13]}$ 是矢量和矩阵的高阶推广。张量的纤维 (fibers) 是类似于矩阵的行和列的术语, 它是只保留 张量一个下标可变而固定其他所有坐标所得到的一 组阵列, 如三阶张量分为模-1 纤维(行纤维)、模-2 纤维(列纤维)和模-3 纤维(管纤维), 分别表示为 $x_{i: k}$,

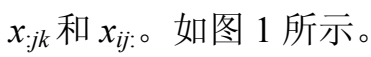

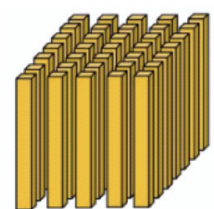

(a) 模 -1 纤维

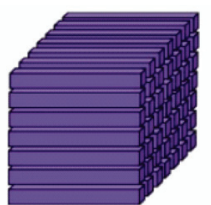

(b) 模 -2 纤维

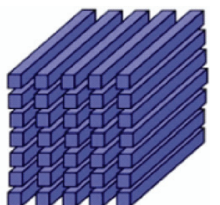

(c) 模 -3 纤维
图 1 三阶张量

除此之外, 张量的切片表示张量的两个维度 部分, 如一个三阶张量会有正面切片、水平切片

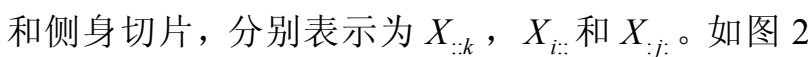
所示。

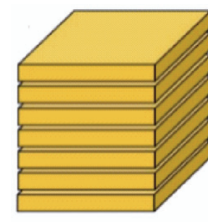

(a) 正面切片

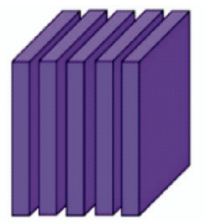

(b) 水平切片

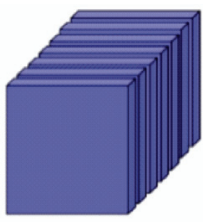

(c) 侧面切片
图 2 三阶张量

张量的内积是由向量的内积扩展而来, 对于两 个大小一致的张量 $\underline{\boldsymbol{A}}, \underline{\boldsymbol{B}} \in \boldsymbol{R}^{I_{1} \times I_{2} \times \ldots I_{N}}$, 它们的内积是 一个标量, 即

$$
(\underline{\boldsymbol{A}}, \underline{\boldsymbol{B}})=\sum_{i_{1}} \sum_{i_{2}} \ldots \sum_{i_{n}} a_{i_{1} \ldots i_{k} \ldots i_{n}} b_{i_{1} \ldots i_{k} \ldots i_{n}}
$$

相应的 Frobenius 范数定义为它的所有元素的 平方和, 张量 $\underline{\boldsymbol{X}} \in \boldsymbol{R}^{I_{1} \times I_{2} \times \ldots I_{N}}$ 则 $\|\underline{X}\|_{F}=\sqrt{(\underline{X}, \underline{X})}$ 。任 何 $1 \leqslant n \leqslant N, n$ - 模张量 $\underline{X} \in \boldsymbol{R}^{I_{1} \times I_{2} \times \ldots I_{N}}$ 与矩阵 $\boldsymbol{M} \in \boldsymbol{R}^{I_{N} \times J}$ 的积, 记为 $\underline{A} \times{ }_{n} M$ 。在展开的矩阵模式 下, 该内积可以表示为

$$
\boldsymbol{y}=\underline{\boldsymbol{A}} \times_{n} \boldsymbol{M} \Leftrightarrow \boldsymbol{Y}_{(n)}=\boldsymbol{M} \boldsymbol{A}_{(n)}
$$

Tucker 分解是矩阵 $\mathrm{SVD}^{[14]}$ 计算的左奇异矩阵或 右奇异矩阵对应的正交子空间在高维空间推广的分 解形式。一个 $N$ 张量 $\underline{\boldsymbol{X}} \in \boldsymbol{R}^{I_{1} \times I_{2} \times \ldots I_{N}}$ 能被写成 Tucker 模型, 如式(3)所示

$$
\underline{\boldsymbol{X}}=\underline{\boldsymbol{G}} \times{ }_{1} A_{1} \times{ }_{2} A_{2} \ldots \times{ }_{n} A_{n}
$$

式中, $\boldsymbol{A}_{i} \in \boldsymbol{R}^{I_{i} \times R_{i}}$ 是因子矩阵, $\underline{\boldsymbol{G}} \in \boldsymbol{R}^{R_{1} \times R_{2} \times \ldots R_{N}}$ 是核 心张量, 代表着张量在各个模的主要成份并且它们 的任何两列都相互正交。Tucker 分解如图 3 所示。
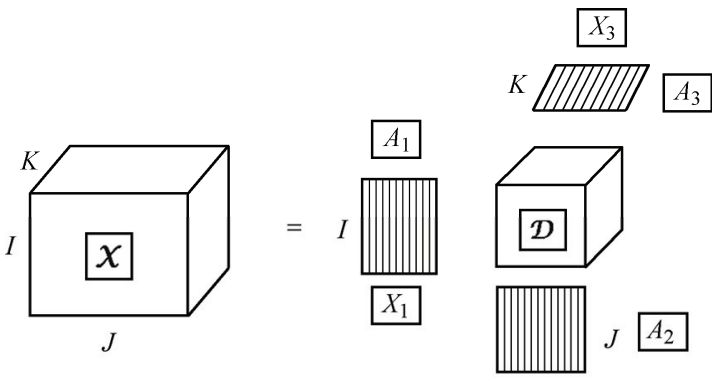

$X_{2}$

图 3 Tucker 分解示意图 


\section{2 基于张量分解的滚动轴承复合故障} 的多通道降噪

\section{1 截断高阶奇异值分解}

截断高阶奇异值分解(Truncated HOSVD)作为一 种正则化方法, 它的基本思想是为了给式(4)定义一个 合适的解，这就是下面提到的一个不适定问题 ${ }^{[15]}$ 。

$$
\min \left(A x-b_{2}^{2}\right)
$$

式中, $\boldsymbol{A}$ 是已知的系数矩阵, $b$ 是包含误差测量结 果的常数, $x$ 是需要求解的变量。不适定问题的解 相对扰动的解是不敏感的。解决这个问题的方法是, 将 $n$ 个奇异值中小于预定阈值的奇异值设置为零。 因此就可以消除小的奇异值对扰动情形下式(4)解 的影响, 消除不适定性质。也就是说, 需要一个低 秩矩阵 $\boldsymbol{A}_{\lambda}$ 去近似原始矩阵 $\boldsymbol{A}$ 。

$$
\left\{\begin{array}{l}
\boldsymbol{A}_{\lambda}=\boldsymbol{U} \boldsymbol{E}_{\lambda} \boldsymbol{V}^{\mathrm{T}} \\
\boldsymbol{E}_{\lambda}=\operatorname{diag}\left(\sigma_{1}, \cdots, \sigma_{\lambda}, 0, \cdots, 0\right)
\end{array}\right.
$$

在这里, $\lambda<n$ 和 $\boldsymbol{E}_{\lambda}$ 是通过取代最小奇异值矩 阵的 $n-\lambda$ 个奇异值为零, 即只考虑较大的前 $\lambda$ 个奇 异值和对应的奇异矢量的影响。之所以采用式(5)定 义矩阵 $\boldsymbol{A}_{\lambda}$ 是因为当 $\lambda$ 选择合适的值时, 矩阵 $\boldsymbol{A}_{\lambda}$ 的 条件数 $\sigma_{1} / \sigma_{\lambda}$ 将更为适中, 从而 Truncated HOSVD 的解将更稳定。相应的 Truncated HOSVD 滤波因子 的形式可以由式(6)表示

$$
\begin{cases}f_{i}=1 & \sigma_{i} \geqslant \sigma_{\lambda} \\ f_{i}=0 & \sigma_{i}<\sigma_{\lambda}\end{cases}
$$

在式 (7) 中通过对矩阵 $\boldsymbol{A}_{\lambda}$ 进行 SVD 得到 Truncated HOSVD 的正则化解

$$
x_{\lambda}=\sum_{i=1}^{n} f_{i} \frac{\mu_{i}^{\mathrm{T}} b}{\sigma_{i}} v_{i}
$$

但是, 它难以确定 Truncated HOSVD 的 $\lambda$ 值。 因此, 尝试采用 $L$ 曲线法确定最佳的正则化参数。

\section{2 正则化参数求解}

$L$ 曲线是由坐标为 $\left(\boldsymbol{A} x_{\lambda}-b_{2}, x_{\lambda}\right)$ 的点构成的一 条曲线, 这些点与正则化参数一一对应, 由于该曲 线通常大体上呈现出 $L$ 形状, 故被称为 $L$ 曲线。 $L$ 曲线法的基本思想是: 对于一个离散的病态问题来 说, 一个合适的选取正则化参数的方法应该同时考 虑正则化解 $\left\|x_{\lambda}\right\|_{2}$ 与残差项 $\left\|A x_{\lambda}-b_{2}\right\|_{2}$ 的大小, 因为 我们希望在正则化解 $\left\|x_{\lambda}\right\|_{2}$ 与残差项 $\left\|\boldsymbol{A} x_{\lambda}-b_{2}\right\|_{2}$ 之间 寻求一个平衡解。 $L$ 曲线的这个角落 $(L$-corner $)$ 对应 于使得正则化解 $\left\|x_{\lambda}\right\|_{2}$ 与残差项 $\left\|\boldsymbol{A} x_{\lambda}-b_{2}\right\|_{2}$ 都较小的
一个均衡解, 由此可以确定这个 L-corner 就对应于 一个较好的正则化参数 ${ }^{[16]}$ 。

\section{3 所提出的方法}

基于张量分解的信号处理方法扩展了低维空间 的信号处理方法。假设多维信号用张量 $\underline{\boldsymbol{T}}$ 表示, 当 其叠加上干扰噪声 $\underline{N}$ 时, 会得到信号, 即被表示为

$$
\underline{R}=\underline{T}+\underline{N}
$$

式中, $\underline{\boldsymbol{R}}, \underline{\boldsymbol{T}}, \underline{\boldsymbol{N}} \in \boldsymbol{R}^{I_{1} \times I_{2} \ldots \times I_{N}}$ 都是 $N$ 阶张量。因此, 在 模型中 $\underline{T}$ 和 $\underline{N}$ 各自表达纯净信号和噪声信号, 使用 多维滤波器估计的无噪声信号可以由式(9)表示 ${ }^{[17]}$

$$
\underline{\hat{\boldsymbol{T}}}=\underline{\boldsymbol{R}} \times{ }_{1} \boldsymbol{H}^{(1)} \times_{2} \ldots \times_{N} \boldsymbol{H}^{(N)}
$$

式中, $\boldsymbol{H}^{(N)}$ 表示一个 $N$ 模滤波器。假设干扰噪声 $\underline{\boldsymbol{N}}$ 与目标信号 $\underline{\boldsymbol{T}}$ 无关, 张量 $\underline{\boldsymbol{R}}$ 的 $n$ 模秩 $K_{n}$ 小于它的 $n$ 模维数。

根据上述张量模型, 假设三阶张量噪声振动信 号 $\underline{\boldsymbol{X}} \in \boldsymbol{R}^{I_{t} \times I_{s} \times I_{c}} ， I_{t}$ 表示时间， $I_{s}$ 表示频率， $I_{c}$ 表示 通道。三阶张量可以用公式表示为

$$
\underline{\boldsymbol{X}}=\underline{\boldsymbol{G}} \times{ }_{1} \boldsymbol{P}_{1} \times{ }_{2} \boldsymbol{P}_{2} \times{ }_{3} \boldsymbol{P}_{3}
$$

$\boldsymbol{P}_{j} \in \boldsymbol{R}^{I_{i} \times R_{i}}$ 是因子矩阵，代表着各个模的重要组成 成分。 $\underline{G} \in \boldsymbol{R}^{R_{1} \times R_{2} \times R_{3}}$ 是核心张量。为了降低信号张 量的维数, 关键是在因子矩阵 $\boldsymbol{P}_{j}$ 中选择适当的左 奇异矢量, 继而很好的保留目标信号, 而不会造成 信号失真。将振动信号构造为三阶张量, 假设每个 因子矩阵中保留的矢量数是 $\lambda_{t} 、 \lambda_{s} 、 \lambda_{c}$ 并且矩阵是 $\boldsymbol{U}^{(1)} 、 \boldsymbol{U}^{(2)} 、 \boldsymbol{U}^{(3)}$ 。因此,一个新的核心张量可以用公 式(11)得到

$$
\underline{\tilde{\boldsymbol{G}}}=\underline{\boldsymbol{X}} \times{ }_{1} \boldsymbol{U}^{(1)^{\mathrm{T}}} \times{ }_{2} \boldsymbol{U}^{(2)^{\mathrm{T}}} \times{ }_{3} \boldsymbol{U}^{(3)^{\mathrm{T}}}
$$

然后, 通过式(12)重构以恢复纯净的目标张量

$$
\underline{\tilde{\boldsymbol{S}}}=\underline{\tilde{\boldsymbol{G}}} \times{ }_{1} \boldsymbol{U}^{(1)} \times{ }_{2} \boldsymbol{U}^{(2)} \times{ }_{3} \boldsymbol{U}^{(3)}
$$
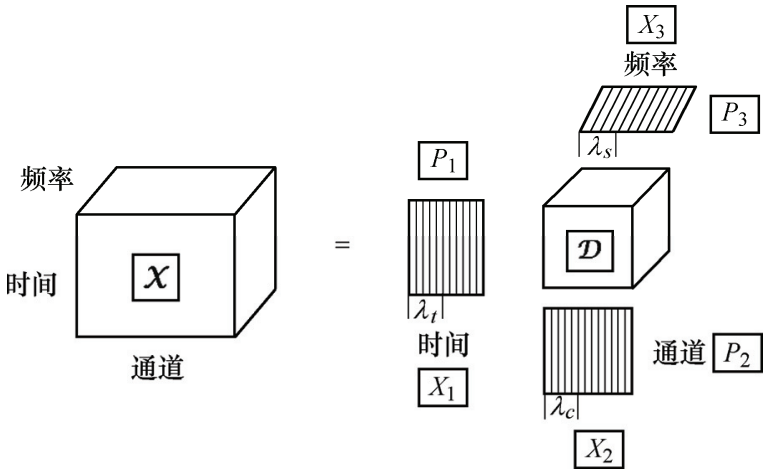

图 4 截断高阶奇异值分解

\section{3 仿真分析}

本文所提出的故障诊断方法框架如图 5 所示。 
为了验证该方法的有效性, 本节模拟了滚动轴承复 合故障信号, 如式(13)所示 ${ }^{[18-19]}$

$$
s_{i}(t)=\sum_{p_{i}=1}^{P_{i}} A_{j} h\left(t-j T_{i}\right)+\delta_{n} n(t)
$$

式中, $P_{i}$ 是脉冲数量, $P_{1}=21, P_{2}=7, P_{3}=5, P_{4}=4$, $P_{5}=12, P_{6}=4, A_{j}$ 是脉冲振幅, 其中 $A_{j}=[0.5,1], T_{i}$ 表示时间周期, 其中 $T_{1}=0.01, T_{2}=0.03, T_{3}=0.05$, $T_{4}=0.06, T_{5}=0.02, T_{6}=0.07$ 。

$h(t)$ 是冲击脉冲函数, $n(t)$ 是一个随机信号。 因为实际信号中的相关特征常常被淹没在噪声中, 所以在脉冲信号中加入高斯白噪声。文中的 $\delta_{n}$ 是噪 声信号的标准差, 反映噪声的强度, 因此, $\delta_{n} h(t)$ 表 示高斯白噪声。 $h(t)$ 能被表示为

$$
\begin{cases}h(t)=\exp (-\beta t) \sin \left(2 \pi f_{r} t\right) & t>0 \\ h(t)=0 & \text { 其他 }\end{cases}
$$

式中, $\beta$ 是一个衰变参数, 其中 $\beta=50 \mathrm{~Hz} ; f_{r}$ 是 共振频频率, 其中 $f_{r}=200 \mathrm{~Hz}$ 。采样频率、时间以 及信号长度分别为 $20 \mathrm{kHz} 、 0.3 \mathrm{~s}$ 和 10000 。六种仿 真信号分别为 $s_{1}(t), s_{2}(t), s_{3}(t), s_{4}(t), s_{5}(t), s_{6}(t)$, 三组复合仿真信号分别为 $\sum_{i=1}^{2} s_{i}(t), \sum_{i=3}^{4} s_{i}(t), \sum_{i=5}^{6} s_{i}(t)$ 。

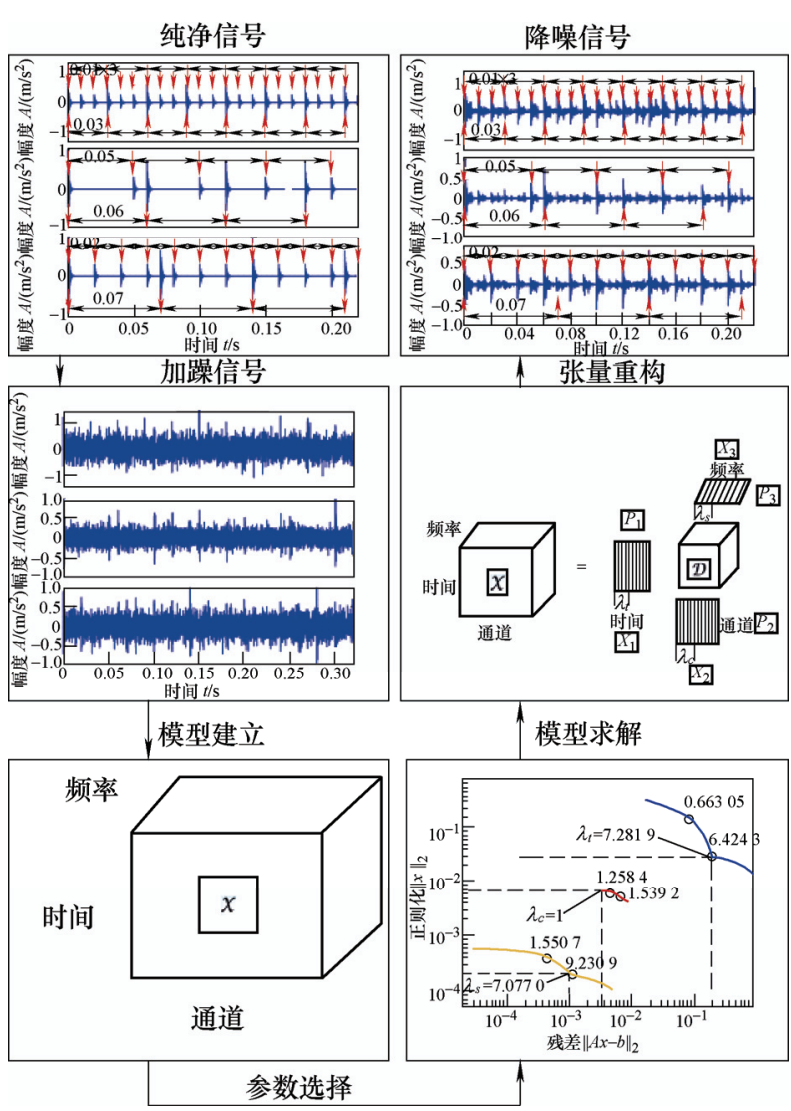

图 5 所提出方法框架图

根据时域信号, 频率和通道将信号建模为一个
三阶张量 $\underline{\boldsymbol{X}} \in \boldsymbol{R}^{300 \times 100 \times 3}$ 。张量分解过程中, 选取张量 的一个正切面的三个行纤维进行分析，根据图 4 中 的 $L$ 曲线求解截断参数, 三个截断参数取整数后分 别为 $\tilde{\lambda}_{t}=7, \tilde{\lambda}_{s}=7, \tilde{\lambda}_{c}=1$ 。则张量分解后这个振动信 号的核心张量为 $\underline{\tilde{\boldsymbol{G}}} \in \boldsymbol{R}^{6 \times 6 \times 2}$, 然后根据核心张量 $\tilde{\boldsymbol{G}}$ 重 新构造目标张量 $\tilde{\boldsymbol{S}}$ 。

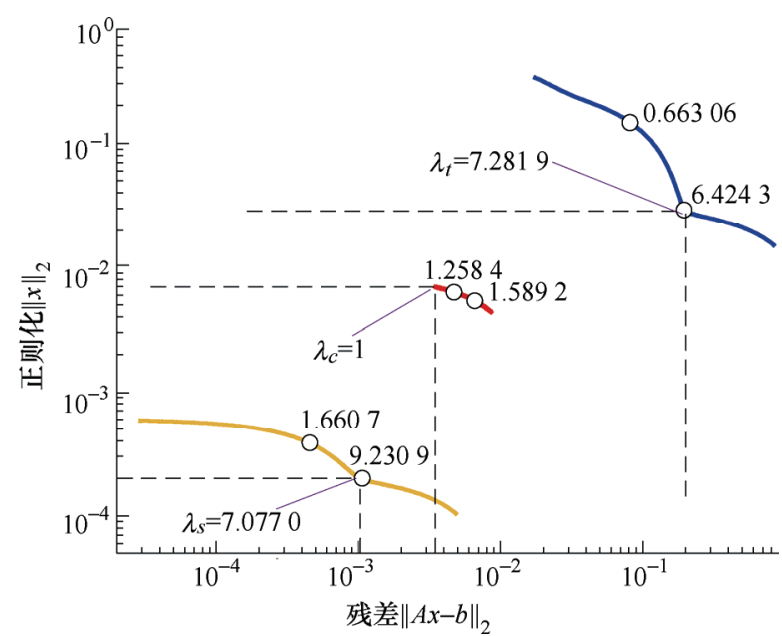

图 6 仿真信号截断参数

图 7a 7c 分别为三个纤维的无噪声信号。三个 纤维增加噪声后的信号如图 $8 \mathrm{a} \sim 8 \mathrm{c}$ 所示。由于噪声 信号干扰, 从图 8 中难以看出脉冲信号的周期特征。 显然从时域上分析原始复合故障信号是无法提取出 轴承故障的特征信息。

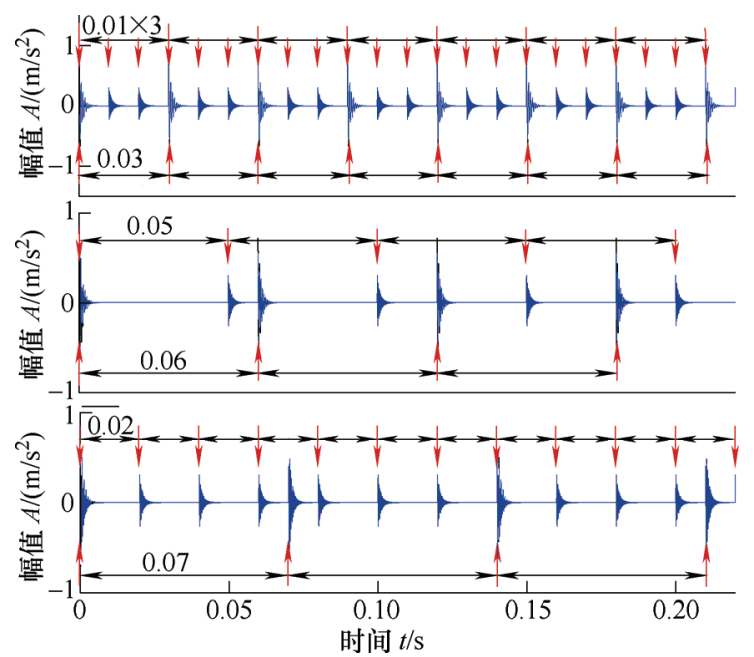

图 7 模-1 纤维无噪声信号

通过本文所提出的方法, 得到降噪后的信号分 别如图 9a 9c 所示。从图 9a 9c 中可以明显地看出 降噪取得了一定的效果, 结果表明能够较好地提取 出脉冲周期特征。通过以上仿真信号的分析, 验证 了基于张量分解的滚动轴承多维降噪技术在复合故 障诊断中的可行性。因此, 基于张量分解的多维降 噪技术可以在后续实例中应用。 


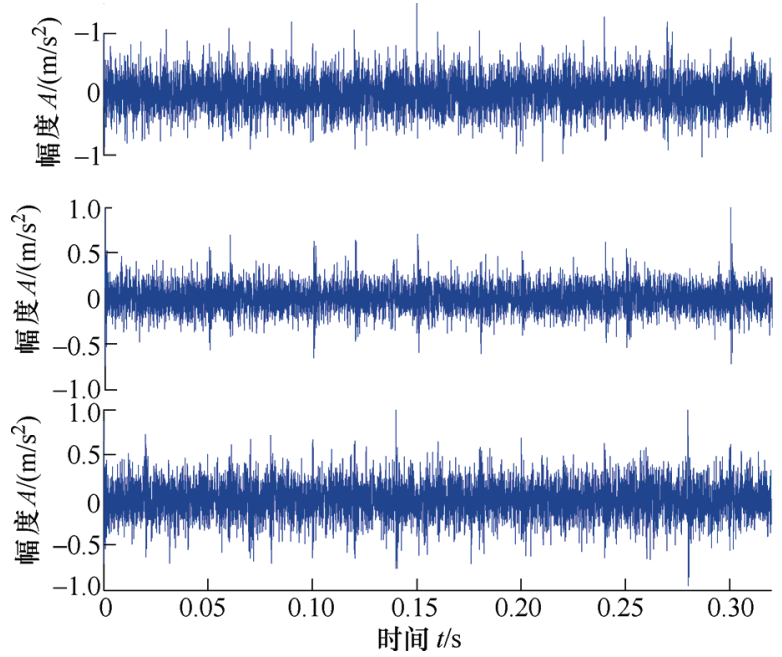

图 8 模-1 纤维噪声信号

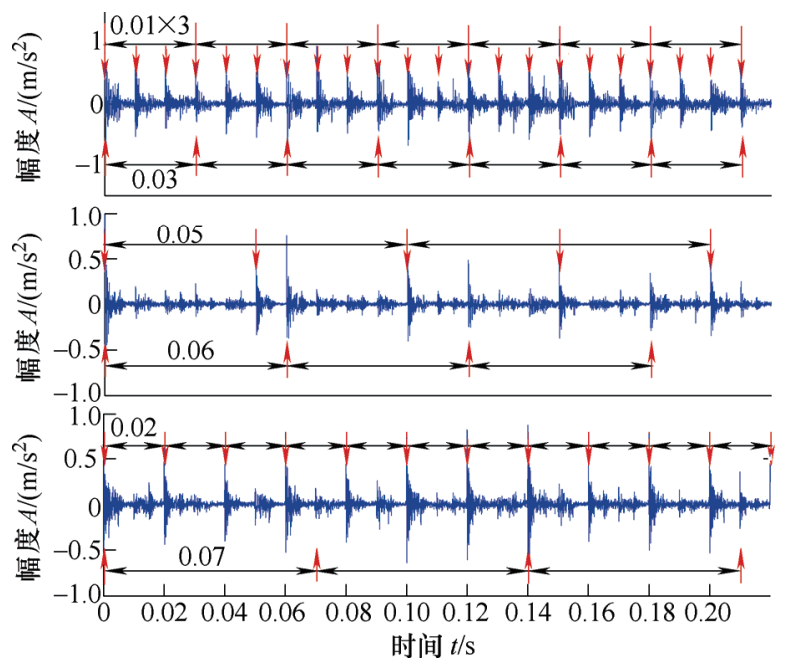

图 9 模-1 纤维纯净信号

\section{4 轴承复合故障诊断实例分析}

为了验证该方法的有效性, 本文利用 MFSMagnum 试验台的试验数据进一步研究了该算法。 试验装置如图 10 所示。试验采用 ER-16K 型轴承, 其规格参数如表 1 所示。采样频率和信号长度分别 为 $20 \mathrm{kHz}$ 和 $10 \mathrm{k}$ 。振动数据是通过安装在轴承底 座附近带加速度计 (灵敏度为 $98 \mathrm{mV} / \mathrm{g}$ ) 的 VQ 数据 采集系统收集的。轴承故障相关参数如表 2 所示。 参考相关资料分别计算试验中使用的外圈轴的故 障频率 BPFO 的理论值和内圈故障频率 BPFI 的理 论值。

表 1 轴承参数

\begin{tabular}{cccc}
\hline 型号 & 节圆直径 $/ \mathrm{mm}$ & 滚动体直径 $/ \mathrm{mm}$ & 滚动体数目 \\
\hline ER-16K & 33.4772 & 7.94 & 8 \\
\hline
\end{tabular}

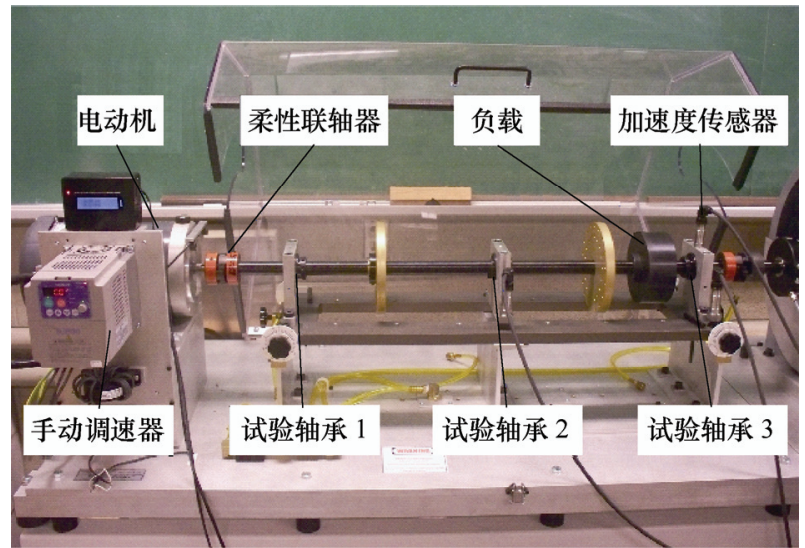

图 10 轴承复合故障检测试验平台

表 2 轴承故障相关参数

\begin{tabular}{cccc}
\hline$f_{r} / \mathrm{Hz}$ & $f_{S} / \mathrm{kHz}$ & $\mathrm{BPFI} / \mathrm{Hz}$ & $\mathrm{BPFO} / \mathrm{Hz}$ \\
\hline 22.23 & 20 & 110.01 & 67.83 \\
22.02 & 20 & 108.97 & 67.19 \\
\hline
\end{tabular}

\section{1 一个轴承的多重故障}

试验过程中, 轴承 $1 、 2 、 3$ 同时具有内圈故 障和外圈故障。输入轴的旋转频率为 $22.02 \mathrm{~Hz}$, 内圈故障频率 $\mathrm{BPFI}=108.97 \mathrm{~Hz}$, 外圈故障频率 $\mathrm{BPFO}=67.19 \mathrm{~Hz}$, 采样频率 $f_{s}$ 设置为 $20 \mathrm{kHz}$ 。根 据时域信号, 频率和通道将振动信号建模为一个三 阶张量 $\underline{X} \in \boldsymbol{R}^{100 \times 300 \times 3}$ 。

在张量分解过程中, 选取张量模型的一个正切 面的三个行纤维进行分析, 根据图 11 中的 $L$ 曲线求 解截断参数。三个截断参数取整数后分别为 $\tilde{\lambda}_{t}=7, \tilde{\lambda}_{s}=8, \tilde{\lambda}_{c}=1$, 则张量分解后这个振动信号的 核心张量为 $\tilde{\boldsymbol{G}} \in \boldsymbol{R}^{6 \times 7 \times 2}$, 然后根据核心张量 $\tilde{\boldsymbol{G}}$ 重新构 造目标张量 $\tilde{\boldsymbol{S}}$ 。

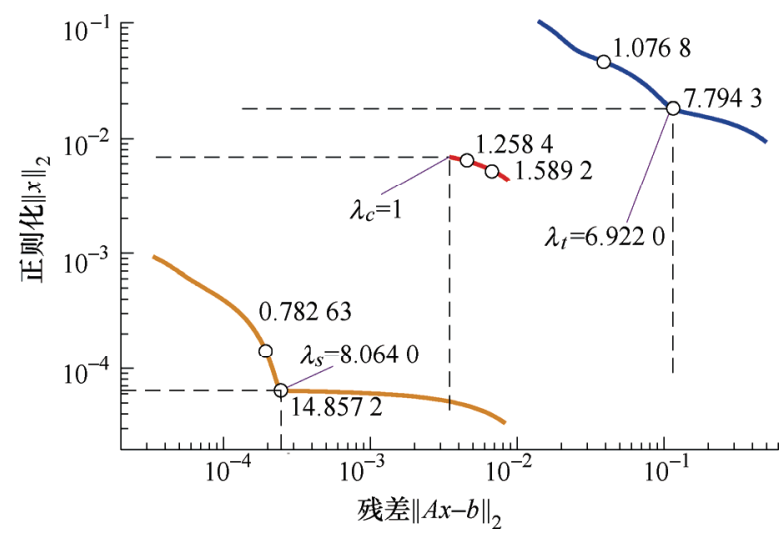

图 11 轴承复合故障截断参数

试验中采集的轴承复合故障振动信号时域波 形分别如图 12a 12c 所示, 然而由于存在较强的 环境噪声, 时域中的特征常常湮没在噪声当中。从 随机噪声和调制现象的时域波形中难以辨别出轴 
承的健康状态以及信号的周期特征。因此, 在时域 中分析原始复合故障信号无法提取出故障的特征 信息。

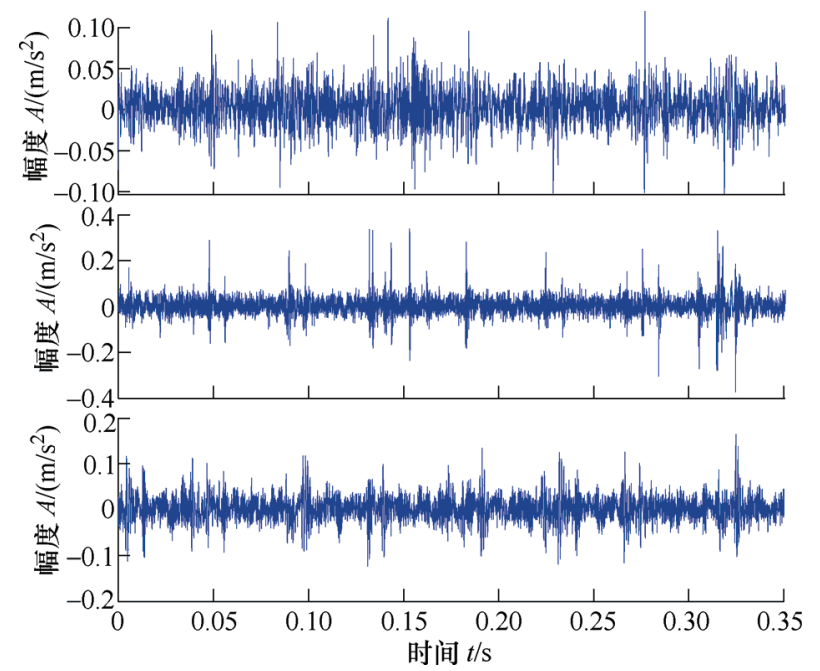

图 12 模-1 纤维原始信号

通过本文所提出的方法得到降噪后的振动信号 如图 13a 13c 所示。与原始信号相比, 在图 13a $13 \mathrm{c}$ 中成功地同时去除了三组振动信号的噪声, 并很 好地保留了脉冲周期特征。从图中可以看出该方法 能够降低噪声并且很大程度地保留了信号的特征结 构。通过同时对复合故障(内圈故障 + 外圈故障)滚动 轴承三组振动信号进行分析, 表明了采用基于张量 分解方法的合理性。同时通过实例分析也验证了本 文所提方法的有效性。

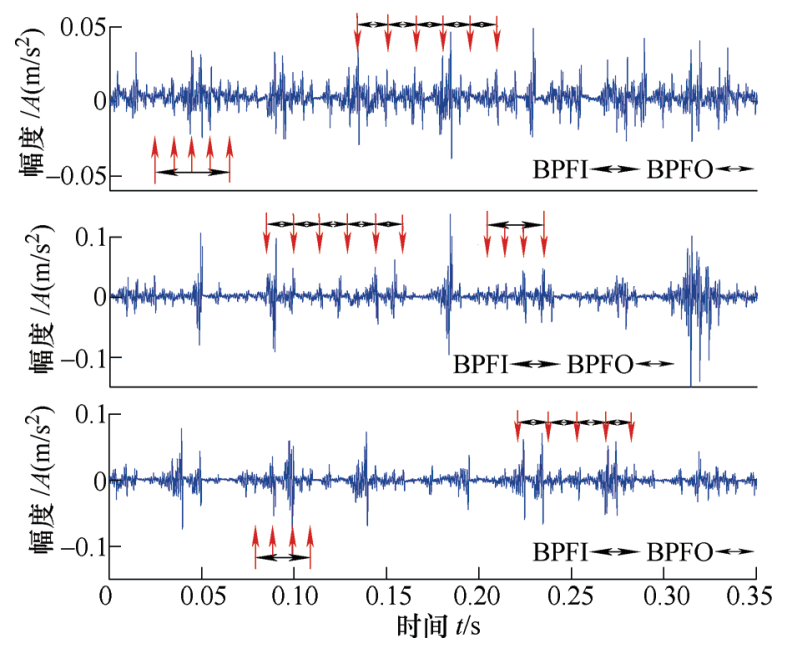

图 13 模-1 纤维纯净信号

\section{2 三个轴承的复合故障}

在试验中, 试验轴承 $1 、 3$ 具有内圈故障, 试 验轴承 2 具有外圈故障。由于滚动轴承存在噪声, 时域中的弱特征常常受到污染。输入轴的旋转频 率为 $22.23 \mathrm{~Hz}$, 内圈故障频率 $\mathrm{BPFI}=110.01 \mathrm{~Hz}$, 外 圈故障频率 $\mathrm{BPFO}=67.83 \mathrm{~Hz}$, 采样频率 $f_{s}$ 设置为
$20 \mathrm{kHz}$ 。

根据时域信号, 频率和通道将振动信号建模为 一个三阶张量 $\boldsymbol{X} \in \boldsymbol{R}^{100 \times 300 \times 3}$ 。在张量分解过程中, 选 取张量模型的一个正切面的三个行纤维进行分析。

根据图 14 中的 $L$ 曲线求解截断参数。三个截断 参数取整数后分别为 $\tilde{\lambda}_{t}=7, \tilde{\lambda}_{s}=6, \tilde{\lambda}_{c}=1$, 则张量分 解后这个振动信号的核心张量为 $\tilde{\boldsymbol{G}} \in \boldsymbol{R}^{6 \times 5 \times 2}$, 然后根 据核心张量 $\tilde{\tilde{\boldsymbol{G}}}$ 重新构造目标张量 $\underline{\tilde{\boldsymbol{S}}}$ 。

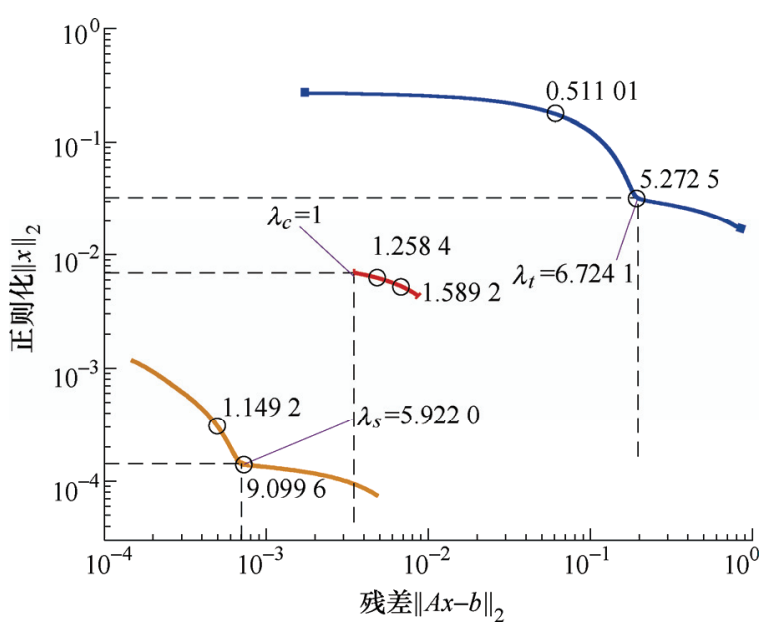

图 14 轴承复合故障截断参数

试验中采集的轴承复合故障振动信号时域波形 如图 $15 \mathrm{a} \sim 15 \mathrm{c}$ 所示, 由于滚动轴承存在缺陷, 振动 信号应呈现脉冲周期特征。然而，由于存在较强的 环境噪声, 时域中的特征容易湮没在噪声当中。从 随机噪声和调制现象的时域波形中难以辨别出轴承 的健康状态。因此, 对原始内、外圈复合故障信号从 时域上进行分析无法提取出轴承故障特征信息。

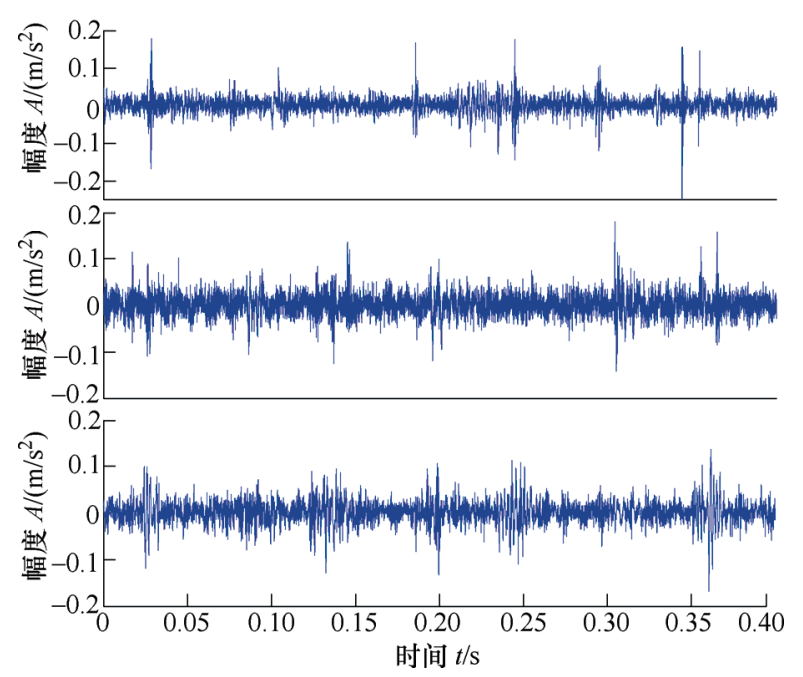

图 15 模-1 纤维原始信号

通过本文提出的方法得到的降噪后的振动信号 如图 16a 16c 所示。与原始信号相比, 在图 16a $16 \mathrm{c}$ 中成功地同时去除了三组振动信号的噪声, 并很 
好地保留了脉冲特征。通过同时对复合故障(内圈故 障+外圈故障)的滚动轴承三组振动信号进行分析, 表明了采用基于张量分解方法的合理性。

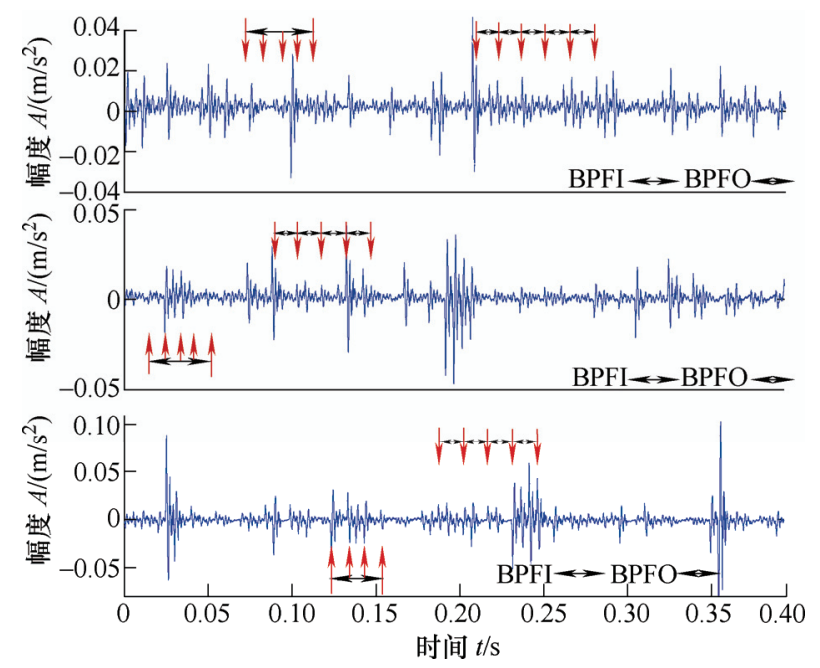

图 16 模-1 纤维纯净信号

本文所提出的方法能够同时将多个通道内的 内、外圈复合故障特征提取出来, 较为理想的实现 了多个通道轴承内、外复合故障特征的提取, 进一 步验证了本文所提出方法的有效性。

\section{5 结论}

本文提出了一种基于张量分析的滚动轴承多通 道复合故障诊断方法, 将振动信号建立为三阶张量, 利用高维空间张量工具解决了振动信号的降噪问 题。通过 Tucker 分解对张量的模纤维进行滤波, 得 到理想的目标张量。采用 $L$ 曲线准则算法求出截断 参数。最后, 通过仿真信号以及两种不同故障模式 的试验对所提出的故障诊断方法的性能进行了全面 的评价, 结果表明了该故障诊断方法可以同时有效 地诊断滚动轴承多个通道的复合故障。

随着大数据的不断快速发展, 将来大数据不可 避免的要在高维空间中进行处理。本文探索研究的 面向大数据的基于张量分析的滚动轴承多通道复合 故障检测方法, 克服了传统方法只能对单通道复合 故障进行诊断的缺陷, 为旋转机械复合故障诊断提 供了一种新方法。我们相信, 各种基于张量分析的 多维信号处理技术将在故障诊断和预测领域成为一 种成功的技术, 特别是将拓宽大数据时代处理异构、 多维数据的视野。

\section{参 考 文 献}

[1] 王建国, 李健, 万旭东. 基于奇异值分解和局域均值分 解的滚动轴承故障特征提取方法 [J]. 机械工程学报,
2015, 51(3): 104-110.

WANG Jianguo, LI Jian, WAN Xudong. Fault feature extraction method of rolling bearings based on singular value decomposition and local mean decomposition[J]. Journal of Mechanical Engneering, 2015, 51(3): 104-110.

[2] WANG Yanxue, HE Zhengjia, ZI Yanyang. Enhancement of signal denoising and multiple fault signatures detecting in rotating machinery using dual-tree complex wavelet transform [J]. Mechanical Systems and Signal Processing, 2010, 24(1): 119-137.

[3] WANG Yanxue, LIANG Ming. An adaptive SK technique and its application for fault detection of rolling element bearings [J]. Mechanical Systems and Signal Processing, 2011, 25 (5): 1750-1764.

[4] LEI Yaguo. Planetary gearbox fault diagnosis using an adaptive stochastic resonance method[J]. Mechanical Systems and Signal Processing, 2013, 38(1): 113-124.

[5] ZHANG Suofeng, WANG Yanxue, HE Shuilong, et al. Bearing fault diagnosis based on variational mode decomposition and total variation denoising[J]. Measurement Science and Technology, 2016, 27(7): 075101.

[6] LI Yifan. Detection of bearing faults using a novel adaptive morphological update lifting wavelet[J]. Chinese Journal of Mechanical Engineering, 2017, 30(6) : 1305-1313.

[7] WANG Yanxue, XIANG Jiawei. Spectral kurtosis for fault detection, diagnosis and prognostics of rotating machines: A review with applications[J]. Mechanical Systems and Signal Processing, 2016, 66(67): 679-698.

[8] WANG Yanxue, WEI Zexian, YANG Jianwei. Feature trend extraction and adaptive density peaks search for intelligent fault diagnosis of machines[J]. IEEE Transactions on Industrial Informatics，2018，15(1): 105-115.

[9] 雷亚国, 贾峰, 孔德同, 等. 大数据下机械智能故障诊 断的机遇与挑战 $[\mathrm{J}]$. 机械工程学报, 2018, 54(5): 94-104. LEI yaguo, JIA Feng, KONG Detong, et al. The opportunity and challenge of mechanical intelligent fault diagnosis under big data[J]. Journal of Mechanical Engineering, 2018, 54(5): 94-104.

[10] ZHANG Yu. Fast nonnegative tensor factorization based on accelerated proximal gradient and low-rank approximation[J]. Neurocomputing, 2016, 198: 148-154.

[11] HAO Zhifeng. A linear support higher-order tensor machine for classification[J]. IEEE Transactions on Image Processing, 2013, 22(7): 2911-2920.

[12] HU Chaofan , WANG Yanxue. Multidimensional denoising of rotating machine based on tensor factorization[J]. Mechanical Systems and Signal Processing, 2019, 122: 273-289. 
[13] KOLDA T G, BADER B W. Tensor decomposition and applications[J]. Siam Review, 2009, 51(3): 455-500.

[14] FIGUEIREDO M, RIBEIRO B, DE ALMEIDA A. Analysis of trends in seasonal electrical energy consumption via non-negative tensor factorization[J]. Neurocomputing, 2015, 170: 318-327.

[15] HANSEN P C. The truncatedsvd as a method for regularization[J]. BIT Numerical Mathematics, 1987, 27(4): 534-553.

[16] REZGHI M, HOSSEINI S M. A new variant of L-curve for Tikhonov regularization[J]. Journal of Computational and Applied Mathematics, 2009, 231(2): 914-924.

[17] MUTI D, BOURENNANE S, MAROT J. Lower-rank tensor approximation and multiway filtering[J]. SIAM
Journal on Matrix Analysis and Applications, 2008(30): 1172-1204.

[18] ERICSSON S. Towards automatic detection of local bearing defects in rotating machines[J]. Mechanical systems and signal processing, 2005, 19(3): 509-535.

[19] WANG Y, LIANG M. Identification of multiple transient faults based on the adaptive spectral kurtosis method[J]. Journal of Sound \& Vibration, 2012，331(2): 470-486.

作者简介: 胡超凡, 男, 1992 年出生, 博士研究生。主要研究方向为动 态信号处理与特征提取与机电装备健康监测。

E-mail: hcf19921230@163.com

王衍学(通信作者), 男, 1980 年出生, 博士, 教授, 博士研究生导师。 主要研究方向为机械系统动力学建模分析, 动态信号处理与特征提取, 装备故障诊断与智能维护。

E-mail: wyx1999140@126.com 